\title{
Correction to: A new approach to a network of congruences on an inverse semigroup
}

\author{
Ying-Ying Feng ${ }^{1} \cdot$ Li-Min Wang $^{2} \cdot$ Lu Zhang $^{2} \cdot$ Hai-Yuan Huang ${ }^{2}$
}

Published online: 3 April 2019

(c) Springer Science+Business Media, LLC, part of Springer Nature 2019

\section{Correction to: Semigroup Forum https://doi.org/10.1007/s00233-019-09993-0}

Article was updated to delete "Petrich" from the following sentence: (11) $\Rightarrow$ (7). Since $\alpha_{n+1} \cap \mathcal{L}=\varepsilon$, by [3, Proposition III.4.2] we have that $\alpha_{n+1}$ is idempotent pure and thus $\alpha_{n+1} \subseteq \tau$.

Publisher's Note Springer Nature remains neutral with regard to jurisdictional claims in published maps and institutional affiliations.

The original article can be found online at https://doi.org/10.1007/s00233-019-09993-0.

$\bowtie \quad$ Li-Min Wang

wanglm@scnu.edu.cn

Ying-Ying Feng

rickyfungyy@fosu.edu.cn

Lu Zhang

262367359@qq.com

Hai-Yuan Huang

243386203@qq.com

1 Department of Mathematics, Foshan University, Foshan 528000, People's Republic of China

2 School of Mathematics, South China Normal University, Guangzhou 510631, People's Republic of China 